\title{
Weight Change, Lifestyle and Mortality in Patients With Type 2 Diabetes
}

Jinbo Hu

Harvard TH Chan School of Public Health https://orcid.org/0000-0003-2925-0583

\section{Yang Hu}

Harvard University HSPH

\section{Ellen Hertzmark}

Harvard University HSPH

\section{Chen Yuan}

Dana-Farber Cancer Institute

\section{Gang Liu}

Harvard University HSPH

Meir J. Stampfer

Harvard University HSPH

\section{Eric B. Rimm}

Harvard University HSPH

Frank B. Hu

Harvard University HSPH

\section{Molin Wang}

Harvard University HSPH

Qi Sun ( $\square$ qisun@hsph.harvard.edu )

Harvard TH Chan School of Public Health

\section{Original investigation}

Keywords: Weight Change, Lifestyle, CVD Mortality, Type 2 Diabetes

Posted Date: August 25th, 2021

DOl: https://doi.org/10.21203/rs.3.rs-826997/v1

License: (1) This work is licensed under a Creative Commons Attribution 4.0 International License. Read Full License 
Version of Record: A version of this preprint was published at The Journal of Clinical Endocrinology \& Metabolism on November 6th, 2021. See the published version at https://doi.org/10.1210/clinem/dgab800. 


\section{Abstract}

Background: Whether weight change around type 2 diabetes (T2D) diagnosis is associated with longterm survival is unclear. We aimed to examine the association between weight change and mortality among participants with incident T2D and evaluate impacts of lifestyle on this association.

Methods: This prospective analysis included 11,262 incident T2D patients from the Nurses' Health Study and Health Professionals Follow-up Study. We assessed weight change bracketing T2D diagnosis in relation to mortality. We also examined potential effect modification by a healthy lifestyle consisting of high-quality diet, regular physical activity, non-smoking status and moderate alcohol consumption.

Results: On average, T2D patients lost $2.3 \mathrm{~kg}$ during a two-year time-window spanning the T2D diagnosis, and body weight increased afterwards. Compared with patients with a stable weight, T2D patients who lost $\geq 10 \%$ body weight had a $26 \%$ (95\% Cl: $4 \%, 52 \%)$ increased mortality due to cardiovascular disease (CVD). Lifestyle significantly modified these associations: the hazard ratios ( $95 \% \mathrm{Cls}$ ) of CVD mortality comparing $\geq 10 \%$ weight loss with stable weight were $1.41(0.87,2.30)$ among participants with a deteriorated lifestyle, $1.46(1.15,1.86)$ for a stable lifestyle, and $0.82(0.53,1.27)$ for an improved lifestyle $\left(P_{\text {interaction }}<0.001\right)$. Major weight loss was also associated with an increased all-cause mortality, and similar effect modifications by lifestyle were observed.

Conclusions: Significant weight loss upon T2D incidence was associated with an increased CVD mortality, although improved lifestyle quality abolished these associations. These results highlight the role of adopting a healthy lifestyle for newly diagnosed T2D patients in improving long-term survival.

\section{Introduction}

In the general population, weight loss lowers blood pressure and improves insulin sensitivity and blood lipids,[1, 2] which are widely-recognized cardiometabolic risk factors. Among patients with newly diagnosed type 2 diabetes (T2D), weight loss is not only associated with better cardiometabolic profiles, $[3,4]$ but also elevated pancreatic $\beta$-cell function,[5] sustained remission of diabetes,[6] and reduced cardiovascular disease (CVD).[7] American Diabetes Association guideline recommends achieving and maintaining $\geq 5 \%$ weight loss in T2D patients. [8] Post-hoc analyses of clinical trials, however, showed that weight loss was independently associated with higher mortality among established T2D patients.[911] Cohort studies in newly diagnosed T2D patients also yielded mixed, contrasting findings of weight loss in relation to mortality.[12-15]

The conflicting evidence for this association may be ascribed, at least partially, to heterogeneity in causes, extent, and timeframe of weight change. Both hyperglycemia and hyperinsulinemia exacerbate protein catabolism and muscle oxidative damage, which gradually result in muscle atrophy and weight loss.[16] As a common symptom of diabetes, weight loss due to the augmented catabolism of protein and muscle, often serves as a marker of disease severity. $[9,16]$ Moreover, studies showed that a modest 
loss of $5 \%$ body weight after diabetes diagnosis was associated with a lower CVD risk,[7] while in longterm T2D patients, significant weight loss (> 10\%) was associated with higher CVD risk and mortality.[9]

To further elucidate this complex and important association, we first described the weight change trajectory since immediately before T2D diagnosis in the Nurses' Health Study (NHS) and the Health Professionals Follow-up Study (HPFS). We then examined the association between weight change starting shortly before diabetes diagnosis and mortality, and lastly evaluated the impact of adopting a healthy lifestyle on this association.

\section{Methods}

\section{Study Population}

The overall description of NHS and HPFS was previously published [17] and shown in Supplementary Methods. The study protocol was approved by the institutional review boards of the Brigham and Women's Hospital and Harvard T.H. Chan School of Public Health. Return of a completed questionnaire was deemed as an informed consent of participation.

The first analysis was to model weight change trajectories among T2D patients from the follow-up questionnaire immediately before diagnosis and throughout the follow-up period after diagnosis. At cohort baseline (1980 for NHS and 1986 for HPFS), we excluded participants with prevalent diabetes, cancer or CVD, those with missing weight assessments, and those age 65 or older to minimize impact by age-related differential muscle loss.[18] We censored follow-up for participants who developed cancer or CVD, died, or reached age 65.

In the analysis of weight change and mortality among T2D patients, only participants with incident T2D were included. Self-reported type 1 diabetes or gestational diabetes cases were excluded. We also excluded participants with existing CVD or cancer at the time of T2D diagnosis. Given the goal of evaluating potential interactions between weight change and lifestyle, we further excluded those who had implausible daily caloric intake ( $<500$ or $>3500 \mathrm{kcal} /$ day for women; $<800$ or $>4200 \mathrm{kcal} /$ day for men), or had missing data on body weight, diet, physical activity, smoking status or alcohol intake on the questionnaire immediately before diabetes diagnosis.

\section{Assessment of Type 2 Diabetes}

Participants who reported T2D diagnosis were sent a supplemental questionnaire that inquired about date of diagnosis, typical symptoms, such as unintended weight loss, diagnostic test results, and hypoglycemic therapy. Based on the questionnaire data, the diagnosis of T2D was ascertained according to the criteria of National Diabetes Data Group (before 1997) or American Diabetes Association (after 1997). $[17,19-21]$ Validation studies showed that $97 \%$ (HPFS) and $98 \%$ (NHS) of questionnaire-confirmed T2D cases were re-confirmed through medical record review.[22, 23] The current analysis is restricted to participants with questionnaire-confirmed T2D. 


\section{Assessment of Body Weight}

Body weight was reported on baseline and follow-up questionnaires. A validation study showed that selfreported weight was highly correlated with measured body weight in NHS and HPFS (Spearman $r=0.97$ in both cohorts).[24]

\section{Assessment of Lifestyle Factors}

A lifestyle score consisting of diet, physical activity, smoking and alcohol consumption was derived to summarize the quality of lifestyle. Quality of diet was assessed by a modified Alternate Healthy Eating Index (AHEI) score without alcohol intake (Supplementary Methods). $[25,26]$ We derived cohort-specific AHEI quintiles, and the top two-fifths were defined as eating a healthy diet. Adequate physical activity was defined as $\geq 150 \mathrm{~min} /$ week of moderate- to vigorous-intensity physical activity. We defined nonsmoking as never or past smoking. Healthy alcohol consumption was defined as a moderate consumption ( 5 to $15 \mathrm{~g} /$ day for women; 5 to $30 \mathrm{~g} /$ day for men).[21]

Participants received 1 point if they met the criterion for the healthy category, and 0 otherwise. The sum of the points across 4 factors constituted a final healthy lifestyle score with a range of 0 to 4 . A higher score indicated a healthier lifestyle. Similar lifestyle scores were strongly associated with lower risk of developing cardiovascular disease or premature deaths in the cohorts.[19, 21]

\section{Assessment of Mortality}

The primary outcome was total and cause specific mortality through 2012 for NHS and 2016 for HPFS. Deaths were identified through searches of the National Death Index, reports by the next of kin, or postal authorities; follow-up for morality was over $98 \%$ complete.[17] The cause of death was determined by physician review of medical records and death certificate information (Supplementary Methods for details).

\section{Statistical Analysis}

We pooled data from the NHS and HPFS to enhance statistical power. In the analysis of weight change trajectories, the goal was to model changes in body weight starting shortly before diagnosis and in subsequent follow-up. We included diabetes-free participants in this analysis for comparison. We set a fixed baseline at 1980 for NHS and 1986 for HPFS for diabetes-free participants, and the baseline for incident diabetes patients was time-varying (Supplementary Methods). We built a multiple generalized linear model which included the baseline calendar year, time since baseline, an interaction variable between time since baseline and diabetes status, and covariates. We used a marginal model of generalized estimating equations (GEE) with independence working variance matrix to calculate the least squares means of weight change at multiple time points in comparison with that at baseline.[27]

For analyses of mortality risk that involved diabetes patient only, person-time accrued from the return of the questionnaire in which diabetes diagnosis was reported until death, or the end of follow-up (2012 for 
NHS and 2016 for HPFS). Weight change was evaluated from the most recent biennial questionnaire before diabetes diagnosis to the first questionnaire after diagnosis. We categorized weight change proportion as major weight loss ( $\leq-10 \%)$, moderate ( $>-10 \%$ to $\leq-5 \%)$, stable weight $(>-5 \%$ to $<5 \%)$, and weight gain $(\geq 5 \%)$. Cox proportional hazards models were used to examine weight changes in relation to mortality with the number of months since the start of follow-up as the time scale. Hazard ratios (HRs) and $95 \%$ confidence intervals (Cls) were calculated in Cox models. Cubic spline regression models were fitted to model potential non-linear associations between weight change and mortality. We used 5 knots $(-0.20,-0.10,-0.05,0.05,0.10)$ to model cubic spline regression, and data outside of $1 \%$ to $99 \%$ percentiles were removed to reduce the impact of outliers.

To explore effect modification of lifestyle on the relationship between weight change and mortality, we stratified the analyses according to lifestyle changes. An improved lifestyle was defined as the healthy lifestyle score at year 2 was larger than the healthy lifestyle score at year 0 . A deteriorated lifestyle was defined as a smaller healthy lifestyle score at year 2 compared to year 0 . Otherwise, the participants' lifestyle quality was considered unchanged. Interactions with lifestyle changes were tested by a likelihood-ratio test comparing models with and without product terms between weight change proportion spline variables and categorized lifestyle changes.

All $P$ values were two-sided, with statistical significance determined as $p<0.05$. Data were analyzed with the use of SAS software, version 9.4 (SAS Institute).

\section{Patient and public involvement}

No patients were involved in setting the research question or the outcome measures, nor were they involved in the design and implementation of the study. We plan to disseminate these findings to participants in our annual newsletter and to the general public in a press release.

\section{Results}

\section{Characteristics of the Study Participants}

Characteristics of participants at baseline are presented in Table 1, stratified by categories of weight change proportion in the time window ( 2 years) between the questionnaires immediately before and after T2D diagnosis. The characteristics by gender were shown in Supplementary Table 1S.

\section{Trajectories of Weight Change}

There were 110,891 diabetes-free participants and 6,494 patients with incident diabetes who met criteria for inclusion in this analysis. Participants without diabetes had an average annual weight gain of $0.3 \mathrm{~kg}$ during 26 years of follow-up. Those with diabetes, on average, lost $2.30 \mathrm{~kg}$ (standard error 0.31 ) during the 2-year time window bracketing diabetes diagnosis, which was followed by a gradual weight gain in a trajectory similar to that among diabetes-free participants (Figure 1). When we used self-reported weight 
at T2D diagnosis in the supplementary questionnaire as baseline weight, we observed a similar pattern of weight changes (Supplementary Figure 1S).

\section{Weight Change and Mortality}

We documented 3,197 deaths in patients with incident T2D, of which 969 were due to CVD, 808 due to cancer, and 1,420 due to other causes. Cubic spline analyses showed a U-shaped relationship between weight change and total mortality with a nadir near zero weight change ( $P$ for curvature $<0.001$ ) (Figure 2A). For deaths due to CVD, cancer or other causes, a non-linear association was also observed (all $P$ for curvature $<0.05$ ). Specifically, weight loss was significantly associated with elevated mortality due to all three causes, although weight gain was significantly associated with deaths due to other causes only (Supplementary Figure 2S).

In categorical analyses of weight change, compared with the stable weight group, major weight loss (>10\%) was associated with a significantly higher mortality (HR 1.21 [95\% Cl 1.09-1.35] for all-cause mortality, 1.26[1.04-1.52] for CVD mortality, 1.24[1.01-1.52] for cancer mortality, 1.19[1.02-1.39] for other causes mortality). Participants with moderate weight loss (5-10\%) or weight gain did not show any increased risk of all-cause mortality or cause-specific mortality (Table 2). In a secondary analysis, when compared with participants who did not report weight loss as a symptom, those who reported this symptom lost more body weight and exhibited a 18\% (95\% Cl: $2 \%, 35 \%)$ higher all-cause mortality and a $37 \%$ (95\% Cl: 6\%, 76\%) higher CVD mortality (Supplementary Table 2S).

\section{Weight Changes, Lifestyle and Mortality}

In cubic spline analyses, the U-shaped relationship between weight change proportion and total mortality was manifested among participants who had a deteriorated or unchanged lifestyle ( $P$ for curvature<0.001), whereas among patients who had improved lifestyle, neither weight loss nor weight gain was significantly associated with mortality ( $P$ for curvature $=0.24)$ (Figure 2B). The interaction between changes of lifestyle and weight change proportion on total mortality was significant $(P<0.001)$.

Similarly, in the categorical analyses, compared with those with stable weight, major weight loss was associated with a $63 \%(95 \% \mathrm{Cl}: 26 \%, 109 \%)$ higher total mortality among T2D patients who had deteriorated lifestyle and $27 \%$ (95\% Cl: $11 \%, 46 \%)$ with unchanged lifestyle. In contrast, among T2D patients who improved their lifestyle, major weight loss was not significantly associated with total mortality (HR[95\% Cl]: 1.02[0.81, 1.27]; $P$ for interaction<0.001) (Table 2). A similar pattern of associations for major weight loss by improved lifestyle also manifested for CVD mortality $(0.82[0.53,1.27])$ or mortality due to other causes $(0.99[0.70,1.40])$. For cancer mortality, none of the associations was significant, though statistical power was limited.

\section{Discussion}


In two large prospective cohort studies, we found a significant weight reduction in the two years around diabetes diagnosis, which was followed by gradual weight gain. Major $(\geq 10 \%)$ weight reduction within two years of diabetes diagnosis was associated with a higher mortality, especially CVD mortality; modest $(<10 \%)$ weight loss or weight gain was not associated with mortality. The elevated mortality associated with major weight loss was primarily observed in T2D patients who had deteriorated or unchanged lifestyle, whereas those who lost $\geq 10 \%$ body weight but improved lifestyle did not exhibit an elevated mortality.

Our observation of the weight trajectory after diabetes diagnosis was consistent with previous studies. Multiple studies have consistently demonstrated a temporary weight loss of approximately $2-3 \mathrm{~kg}$ within 1-2 years after diabetes diagnosis,[28-30] and a gradual weight gain afterwards.[28] The current study evaluated weight change trajectory during an extended 26-year follow-up and also compared the trajectory with that among diabetes-free participants in the same cohorts. Overall, these data suggest that weight loss is commonly observed in diabetes patients, although this weight loss is transient and confined to the 1-2 years period proximate to disease diagnosis.

The long-term health consequences of weight changes after diabetes diagnosis have been examined in previous studies, but results were somewhat inconsistent. Some,[12] but not all studies,[7, 15] showed that a significant weight loss after diabetes diagnosis was associated with a high mortality. It is interesting to note that among patients with established diabetes history, major weight loss was more consistently associated with adverse CVD outcomes.[9-11] The reason for the inconsistent findings in previous studies is unknown, although differences in the time-window of weight change assessment, baseline disease duration and severity, and other serious illnesses that could induce weight loss may likely account for some of the mixed findings. With data collected during 30 years of follow-up in the NHS and HPFS, we were able to, for the first time, examine weight change in the time window that brackets the diabetes incidence in relation to mortality. Elevated mortality due to CVD, cancer, or other causes was observed primarily among T2D patients who lost significant body weight, but not among patients in other categories of weight change.

Weight loss upon diabetes diagnosis is mainly accounted by the loss of lean mass. In the Health, Aging, and Body Composition (Health $A B C$ ) study, newly-diagnosed T2D patients lost more lean mass than established T2D patients and non-diabetes individuals $(-186 \pm 25,-106 \pm 20$, and $-125 \pm 7 \mathrm{~g} / \mathrm{year}$, respectively), while the changes of total fat mass were similar among the groups.[31] Adequate lean mass is consistently associated with lower mortality among populations with or without diabetes.[32, 33] In addition, major weight loss in newly-onset T2D may serve as a marker of disease severity as this symptom is often correlated with chronic kidney diseases, ischemic heart disease and stroke.[34] Future studies are warranted to further illustrate these and other mechanisms underlying the associations between this transient weight loss and adverse health consequences.

A healthy lifestyle is critical for preserving adequate lean mass during weight loss.[16] In addition, multiple lines of evidence have suggested that a healthy lifestyle may improve cardiometabolic health 
with and without promoting weight loss.[21, 35-37] In the current analysis, improved lifestyle largely abolished the positive association between weight loss and mortality. Previous studies showed improved cardiometabolic health and lowered mortality among T2D patients who practiced healthy lifestyles.[21, 38] Of note, in the current analysis, we were unable to distinguish between intentional and unintentional weight loss. However, T2D patients who reported having unintentional weight loss as a symptom were more likely to lose $\geq 10 \%$ body weight $(23.3 \%$ vs. $13.6 \%)$ and this symptom was associated with a similar elevation of mortality, suggesting that the weight loss was largely unintentional in the current analysis. Collectively, the current study, together with evidence from studies that examined intentional weight loss, $[7,37,39]$ highlighted the role of a healthy lifestyle in improving the survival of diabetes patients. These results also implied that adopting a healthy lifestyle might be more important than emphasizing on weight loss alone.

The strengths of our study include the long term follow-up of two large cohorts with low rates of loss to follow-up, and the comprehensive, repeated assessments of body weight and lifestyle factors. Our study has some limitations. First, the questionnaire administration was not exactly aligned with the diabetes diagnosis. However, the biennial questionnaire enabled us to assess body weight before the diabetes incidence and thus the weight change that occurred from shortly before to after diabetes diagnosis, which was typically not captured in studies of patients with existing diabetes. Moreover, using body weight at diabetes diagnosis in a sensitivity analysis, we found similar association between significant weight loss and mortality. Second, although we adjusted for multiple confounders, we cannot exclude the possibility that these associations may still be partially ascribed to residual or unmeasured confounding. Finally, more than $95 \%$ of our participants were Caucasians and all of them were registered nurses or health professionals. The homogeneity is helpful for controlling for confounding, although our findings need to be replicated in other populations with different characteristics.

\section{Conclusion}

In conclusion, we showed a transient weight loss surrounding the period of T2D diagnosis. During this period, a significant weight loss was associated with higher total and CVD mortality particularly among patients who had a deteriorated or unchanged lifestyle, whereas among patients who adopted a healthy lifestyle the elevated mortality was largely attenuated. These findings emphasize the importance of healthy lifestyle in improving the long-term survival of diabetes patients.

\section{Declarations}

Acknowledgement: We thank the participants and staff of the Nurses' Health Study and the Health Professionals Follow-Up Study for their valuable contributions as well as the following state cancer registries for their help: AL, AZ, AR, CA, CO, CT, DE, FL, GA, ID, IL, IN, IA, KY, LA, ME, MD, MA, MI, NE, NH, NJ, NY, NC, ND, OH, OK, OR, PA, RI, SC, TN, TX, VA, WA, WY. The authors assume full responsibility for analyses and interpretation of these data. 
Contributors: QS, JBH and YH participated in project conception and development of research methods; QS, FBH and EBR obtained funding and provided oversight; JBH, YH, EH, CY, GL, MW and QS analyzed data and performed analysis; JBH and QS drafted the article; QS, YH, MJS, FBH and EBR contributed to data interpretation and critical review of the report.

Funding: This study was funded by the US National Institutes of Health, grant numbers CA186107, CA176726, CA167552, DK120870, DK082486, HL35464, HL088521, DK058845, U01 CA167552, and HL034594.

Competing interests: All authors have completed the ICMJE uniform disclosure form at www.icmje.org/coi_disclosure.pdf and declare: support from the National Institutes of Health for the submitted work. All authors report no relationship or activity that could appear to have influenced the submitted work.

Ethical approval: The Nurses' Health Study and the Health Professionals Follow-up Study were approved by the institutional review boards at Brigham and Women's Hospital and Harvard T.H. Chan School of Public Health.

Data sharing: No additional data available.

Transparency statement: The lead author (JBH) affirms that the manuscript is an honest, accurate, and transparent account of the study being reported; that no important aspects of the study have been omitted; and that any discrepancies from the study as planned (and, if relevant, registered) have been explained.

Dissemination to participants and related patient and public communities: There is no plan to disseminate the results of the research to study participants or the relevant patient community. The participants are updated on the study outcomes and developments through the study websites (www.nurseshealthstudy.org and www. hsph.harvard.edu/hpfs) and newsletters.

\section{Availability of data and materials}

The datasets used and/or analyzed in the current study are available from the corresponding author upon reasonable request.

\section{Consent for publication}

Not applicable.

\section{References}

1. Zomer E, Gurusamy K, Leach R, Trimmer C, Lobstein T, Morris S, James WP, Finer N: Interventions that cause weight loss and the impact on cardiovascular risk factors: a systematic review and meta-analysis. Obes Rev 2016, 17(10):1001-1011. 
2. Ades PA, Savage PD: Potential benefits of weight loss in coronary heart disease. Prog CardiovasC Dis 2014, 56(4):448-456.

3. Feldstein AC, Nichols GA, Smith DH, Stevens VJ, Bachman K, Rosales AG, Perrin N: Weight Change in Diabetes and Glycemic and Blood Pressure Control. Diabetes Care 2008, 31(10):1960-1965.

4. Patel YR, Kirkman MS, Considine RV, Hannon TS, Mather KJ: Changes in Weight and Glucose Can Protect Against Progression in Early Diabetes Independent of Improvements in beta-Cell Function. J Clin Endocrinol Metab 2016, 101(11):4076-4084.

5. Solomon TP, Haus JM, Kelly KR, Rocco M, Kashyap SR, Kirwan JP: Improved pancreatic beta-cell function in type 2 diabetic patients after lifestyle-induced weight loss is related to glucose-dependent insulinotropic polypeptide. Diabetes Care 2010, 33(7):1561-1566.

6. Dambha-Miller H, Day AJ, Strelitz J, Irving G, Griffin SJ: Behaviour change, weight loss and remission of Type 2 diabetes: a community-based prospective cohort study. Diabet Med 2020, 37(4):681-688.

7. Strelitz J, Ahern AL, Long GH, Hare MJL, Irving G, Boothby CE, Wareham NJ, Griffin SJ: Moderate weight change following diabetes diagnosis and 10 year incidence of cardiovascular disease and mortality. Diabetologia 2019, 62(8):1391-1402.

8. 8. Obesity Management for the Treatment of Type 2 Diabetes: Standards of Medical Care in Diabetes2021. Diabetes Care 2021, 44(Suppl 1):S100-S110.

9. Lee AK, Woodward M, Wang D, Ohkuma T, Warren B, Sharrett AR, Williams B, Marre M, Hamet P, Harrap $S$ et al: The Risks of Cardiovascular Disease and Mortality Following Weight Change in Adults with Diabetes: Results from ADVANCE. J Clin Endocrinol Metab 2020, 105(1).

10. Doehner W, Gerstein HC, Ried J, Jung H, Asbrand C, Hess S, Anker SD: Obesity and weight loss are inversely related to mortality and cardiovascular outcome in prediabetes and type 2 diabetes: data from the ORIGIN trial. Eur Heart J 2020, 41(28):2668-2677.

11. Yeboah P, Hsu FC, Bertoni AG, Yeboah J: Body Mass Index, Change in Weight, Body Weight Variability and Outcomes in Type 2 Diabetes Mellitus (from the ACCORD Trial). Am J Cardiol 2019, 123(4):576-581.

12. Kim MK, Han K, Koh ES, Kim ES, Lee M-K, Nam GE, Kwon H-S: Weight change and mortality and cardiovascular outcomes in patients with new-onset diabetes mellitus: a nationwide cohort study. Cardiovasc Diabetol 2019, 18(1).

13. Owusu ESA, Samanta M, Shaw JE, Majeed A, Khunti K, Paul SK: Weight loss and mortality risk in patients with different adiposity at diagnosis of type 2 diabetes: a longitudinal cohort study. Nutr Diabetes 2018, 8(1):37. 
14. Koster-Rasmussen R, Simonsen MK, Siersma V, Henriksen JE, Heitmann BL, de Fine Olivarius N: Intentional Weight Loss and Longevity in Overweight Patients with Type 2 Diabetes: A Population-Based Cohort Study. PLoS One 2016, 11(1):e0146889.

15. Bodegard J, Sundstrom J, Svennblad B, Ostgren CJ, Nilsson PM, Johansson G: Changes in body mass index following newly diagnosed type 2 diabetes and risk of cardiovascular mortality: a cohort study of $\mathbf{8 4 8 6}$ primary-care patients. Diabetes Metab 2013, 39(4):306-313.

16. Kalyani RR, Corriere M, Ferrucci L: Age-related and disease-related muscle loss: the effect of diabetes, obesity, and other diseases. Lancet Diabetes Endocrinol 2014, 2(10):819-829.

17. Tobias DK, Pan A, Jackson CL, O'Reilly EJ, Ding EL, Willett WC, Manson JE, Hu FB: Body-mass index and mortality among adults with incident type 2 diabetes. N Engl J Med 2014, 370(3):233-244.

18. Mozaffarian D, Hao T, Rimm EB, Willett WC, Hu FB: Changes in diet and lifestyle and long-term weight gain in women and men. N Engl J Med 2011, 364(25):2392-2404.

19. Li Y, Schoufour J, Wang DD, Dhana K, Pan A, Liu X, Song M, Liu G, Shin HJ, Sun Q et al: Healthy lifestyle and life expectancy free of cancer, cardiovascular disease, and type 2 diabetes: prospective cohort study. BMJ 2020, 368:16669.

20. Hu Y, Zong G, Liu G, Wang M, Rosner B, Pan A, Willett WC, Manson JE, Hu FB, Sun Q: Smoking Cessation, Weight Change, Type 2 Diabetes, and Mortality. N Engl J Med 2018, 379(7):623-632.

21. Liu G, Li Y, Hu Y, Zong G, Li S, Rimm EB, Hu FB, Manson JE, Rexrode KM, Shin HJ et al: Influence of Lifestyle on Incident Cardiovascular Disease and Mortality in Patients With Diabetes Mellitus. J Am Coll Cardio/2018, 71(25):2867-2876.

22. Hu FB, Leitzmann MF, Stampfer MJ, Colditz GA, Willett WC, Rimm EB: Physical activity and television watching in relation to risk for type 2 diabetes mellitus in men. Arch Intern Med 2001, 161(12):1542-1548.

23. Manson JE, Rimm EB, Stampfer MJ, Colditz GA, Willett WC, Krolewski AS, Rosner B, Hennekens CH, Speizer FE: Physical activity and incidence of non-insulin-dependent diabetes mellitus in women. Lancet 1991, 338(8770):774-778.

24. Rimm EB, Stampfer MJ, Colditz GA, Chute CG, Litin LB, Willett WC: Validity of self-reported waist and hip circumferences in men and women. Epidemiology 1990, 1(6):466-473.

25. Chiuve SE, Fung TT, Rimm EB, Hu FB, McCullough ML, Wang M, Stampfer MJ, Willett WC: Alternative dietary indices both strongly predict risk of chronic disease. J Nutr 2012, 142(6):1009-1018.

26. Kennedy ET, Ohls J, Carlson S, Fleming K: The Healthy Eating Index: design and applications. J Am Diet Assoc 1995, 95(10):1103-1108. 
27. Pepe MS, Anderson GL: A cautionary note on inference for marginal regression models with longitudinal data and general correlated response data. Communications in Statistics - Simulation and Computation 2007, 23(4).

28. Looker HC, Knowler WC, Hanson RL: Changes in BMI and weight before and after the development of type 2 diabetes. Diabetes Care 2001, 24(11):1917-1922.

29. de Fine Olivarius N, Andreasen AH, Siersma V, Richelsen B, Beck-Nielsen H: Changes in patient weight and the impact of antidiabetic therapy during the first 5 years after diagnosis of diabetes mellitus. Diabetologia 2006, 49(9):2058-2067.

30. de Fine Olivarius N, Siersma VD, Koster-Rasmussen R, Heitmann BL, Waldorff FB: Weight changes following the diagnosis of type 2 diabetes: the impact of recent and past weight history before diagnosis. results from the Danish Diabetes Care in General Practice (DCGP) study. PLoS One 2015, 10(4):e0122219.

31. Park SW, Goodpaster BH, Lee JS, Kuller LH, Boudreau R, de Rekeneire N, Harris TB, Kritchevsky S, Tylavsky FA, Nevitt $\mathrm{M}$ et al: Excessive loss of skeletal muscle mass in older adults with type 2 diabetes. Diabetes Care 2009, 32(11):1993-1997.

32. Lee DH, Keum N, Hu FB, Orav EJ, Rimm EB, Willett WC, Giovannucci EL: Predicted lean body mass, fat mass, and all cause and cause specific mortality in men: prospective US cohort study. BMJ 2018, 362:k2575.

33. Murea M, Lenchik L, Register TC, Russell GB, Xu J, Smith SC, Bowden DW, Divers J, Freedman BI: Psoas and paraspinous muscle index as a predictor of mortality in African American men with type 2 diabetes mellitus. J Diabetes Complications 2018, 32(6):558-564.

34. Nam GE, Park YG, Han K, Kim MK, Koh ES, Kim ES, Lee MK, Kim B, Hong OK, Kwon HS: BMI, Weight Change, and Dementia Risk in Patients With New-Onset Type 2 Diabetes: A Nationwide Cohort Study. Diabetes Care 2019, 42(7):1217-1224.

35. Liu G, Hu Y, Zong G, Pan A, Manson JE, Rexrode KM, Rimm EB, Hu FB, Sun Q: Smoking cessation and weight change in relation to cardiovascular disease incidence and mortality in people with type 2 diabetes: a population-based cohort study. Lancet Diabetes Endocrinol 2020, 8(2):125-133.

36. Jiao J, Liu G, Shin HJ, Hu FB, Rimm EB, Rexrode KM, Manson JE, Zong G, Sun Q: Dietary fats and mortality among patients with type 2 diabetes: analysis in two population based cohort studies. BMJ 2019, 366:14009.

37. Gregg EW, Jakicic JM, Blackburn G, Bloomquist P, Bray GA, Clark JM, Coday M, Curtis JM, Egan C, Evans $M$ et al: Association of the magnitude of weight loss and changes in physical fitness with long- 
term cardiovascular disease outcomes in overweight or obese people with type 2 diabetes: a post-hoc analysis of the Look AHEAD randomised clinical trial. Lancet Diabetes Endocrino/2016, 4(11):913-921.

38. American Diabetes A: 5. Lifestyle Management: Standards of Medical Care in Diabetes-2019. Diabetes Care 2019, 42(Suppl 1):S46-S60.

39. Griffin SJ, Borch-Johnsen K, Davies MJ, Khunti K, Rutten GE, Sandbaek A, Sharp SJ, Simmons RK, van den Donk M, Wareham NJ et al: Effect of early intensive multifactorial therapy on 5-year cardiovascular outcomes in individuals with type 2 diabetes detected by screening (ADDITION-Europe): a clusterrandomised trial. Lancet 2011, 378(9786):156-167.

\section{Tables}

Due to technical limitations, table 1-2 is only available as a download in the Supplemental Files section.

\section{Figures}

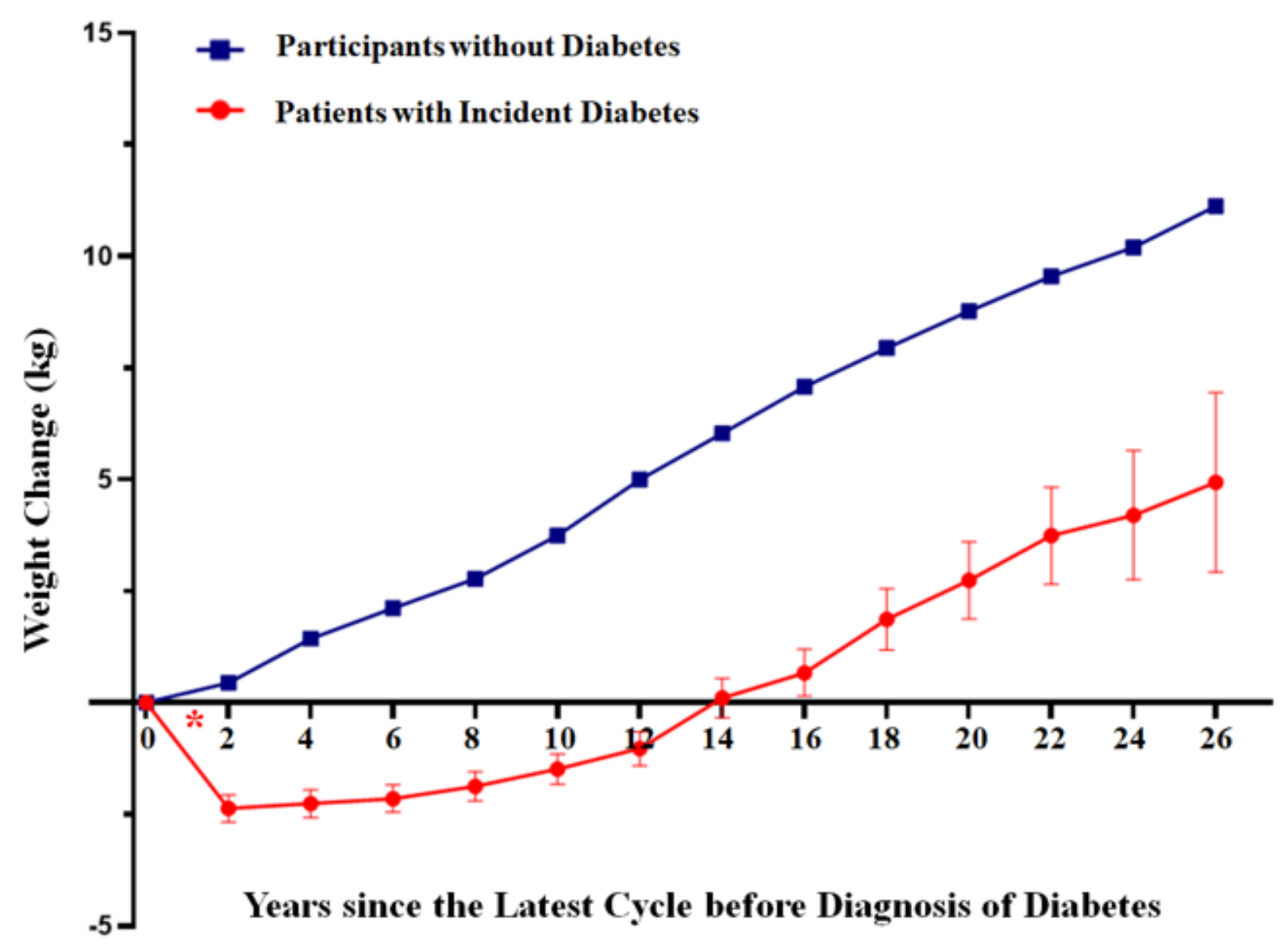

Figure 1 
Trajectories of Weight Change among Those with Incident Diabetes since the Most Recent Biennial Questionnaire before Diabetes Diagnosis, Compared to Participants without Diabetes since the Cohort Baseline. In the group of incident diabetes, the red asterisk ' $*$ ' indicates the time frame of diabetes onset. Body weight at the time-point ' 0 ' was defined as the most recent weight before diabetes diagnosis, and body weight at time-point ' 2 ' was recorded on the first biennial questionnaire after diabetes diagnosis; and so on. In participants without diabetes, body weight at the time-point ' 0 ' was defined as the cohort baseline (1980 for NHS and 1986 for HPFS), and the time-point ' 2 ' was 2 years after baseline, and so on. Weight changes were truncated within -50 to $50 \mathrm{~kg}$. Participants were censored at age 65 . We used a marginal model of generalized estimating equations with independence working variance matrix to calculate the least squares means of weight change at multiple time points in comparison with that at year 0 . The model included the baseline calendar year, time since baseline, an interaction variable between time since baseline and diabetes status, and other covariates. We stopped updating the trajectory since the number of participants at follow-up decreased to $<1 \%$ of the baseline in diabetes group. Values of weight change are mean \pm standard error. 

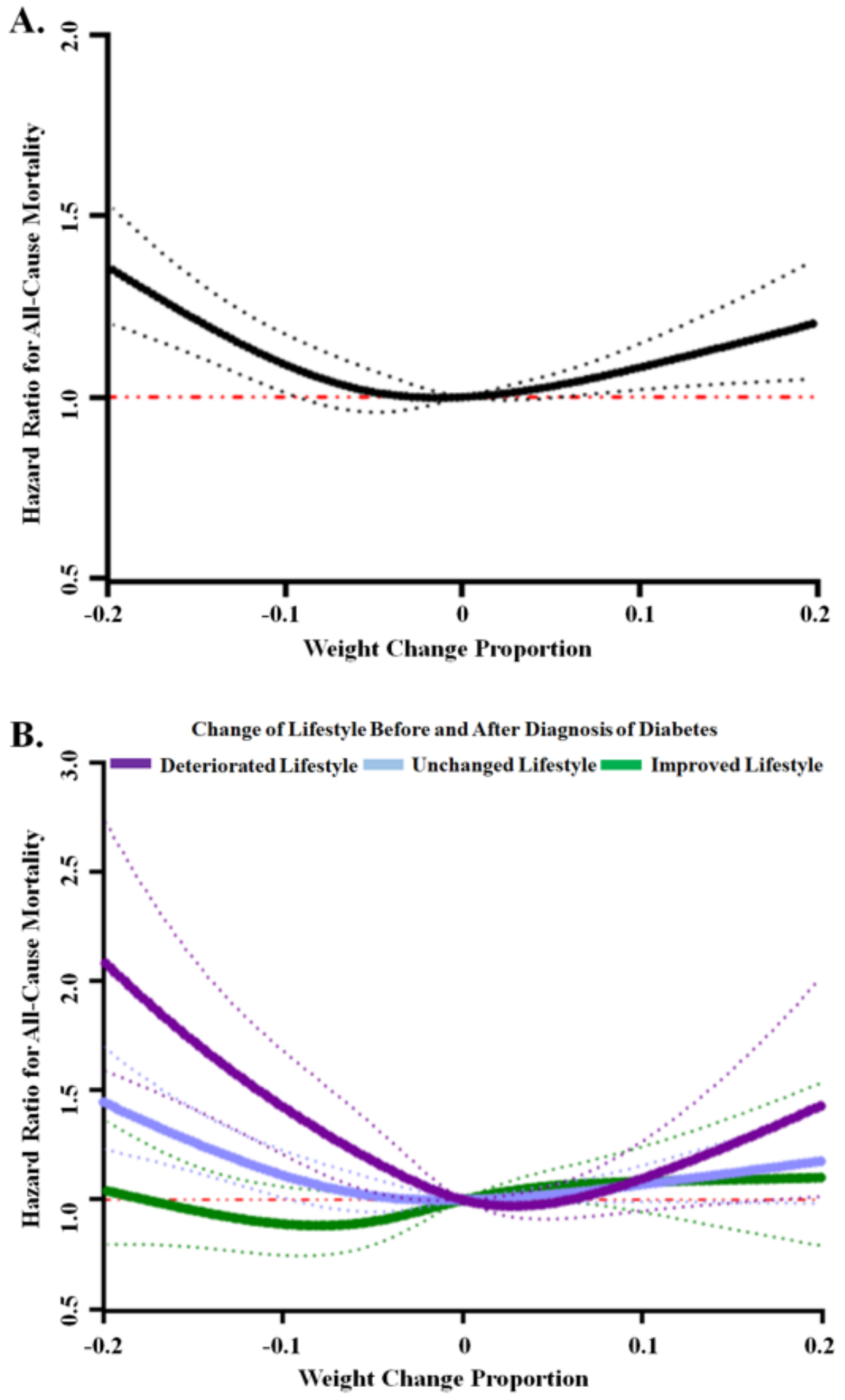

Figure 2

Associations between Weight Change Proportion and Risk of All-Cause Mortality, according to Changes of Lifestyle before and after Diabetes Diagnosis. Panel A shows the overall relationship between weight change proportion and all-cause mortality ( $P$ for curvature $<0.001)$. Panel $B$ shows the relationship according to changes of lifestyle before and after diabetes diagnosis. In subgroups of deteriorated,unchanged, improved lifestyle, $P$ values for curvature were $<0.001,<0.001$ and 0.244 , 
respectively. Changes in weight and lifestyle were evaluated from the most recent biennial questionnaire before diabetes diagnosis to the first questionnaire after diagnosis. The weight change proportion was calculated as ([weight after diagnosis] - [weight before diagnosis]) / [weight before diagnosis]. All of these associations were based on a multivariate model which adjusted for age, sex, ethnicity, history of hypertension, current antidiabetic medication use, current antihypertensive medication use, hypercholesterolemia, current anti-hypercholesterolemia medication use in the questionnaire cycle right after diabetes diagnosis, family history of diabetes, family history of myocardial infarction, current aspirin use, current multivitamin use, diabetes duration and lifestyle score before diabetes diagnosis. Dotted lines represent $95 \%$ confidence intervals.

\section{Supplementary Files}

This is a list of supplementary files associated with this preprint. Click to download.

- 8.18.SupplementQS.docx

- Tables.pdf 